\title{
Critérios de Construção e Relato da Análise Prototípica para Representações Sociais
}

\author{
João Wachelke \\ Università degli studi di Padova \\ Rafael Wolter \\ Universidade do Estado do Rio de Janeiro
}

\begin{abstract}
RESUMO - A análise prototípica é uma convenção de apresentação bastante difundida para caracterizar a estrutura de uma representação social a partir de dados de evocações livres. O presente texto visa a sistematizar e indicar algumas dessas informações que deveriam estar presentes na descrição de resultados da análise prototípica, discutindo brevemente os prós e contras de algumas opções de realização da análise. Para tanto, é feita uma breve introdução da análise e posteriormente passa-se a considerações técnicas, finalizando com um exemplo de relato.
\end{abstract}

Palavras-chave: análise prototípica; análise de evocações; associações livres; representações sociais.

\section{Criteria Related to the Realization and Reporting of Prototypical Analysis for Social Representations}

\begin{abstract}
Prototypical analysis is a largely diffused convention of presentation to characterize the structure of a social representation based on free association data. However, occasionaly the results of that analysis do not contain the essential information to guarantee its transparency. The present text aims at systematizing and indicating some of the information that should be present in the description of prototypical analysis results, briefly discussing the pros and cons of some analysis options. For that purpose, a brief introduction of the analysis is made, then moving on to technical considerations and closing the text with an example of an report.
\end{abstract}

Keywords: prototypical analysis; evocation analysis; free associations; social representations.

A abordagem estrutural é uma escola dentre as diversas existentes para o estudo do fenômeno das representações sociais. Trata-se de uma perspectiva que concebe representações sociais como estruturas de conhecimento sobre temas da vida social, compartilhadas por grupos e formadas por elementos cognitivos ligados entre si. A principal teoria da abordagem estrutural é a teoria do núcleo central, que defende que as representações sociais são um duplo sistema formado por dois tipos de elementos: um núcleo central e o sistema periférico. O núcleo é composto por um conjunto restrito de elementos que define a representação social e a organiza; é fortemente compartilhado pelo grupo. O sistema periférico compreende a maior parte dos elementos da representação, os quais possuem natureza condicional e têm caráter mais flexível e prático, adaptando a representação às experiências cotidianas (Sá, 1996; Flament \& Rouquette, 2003).

A análise prototípica (também chamada análise de evocações ou das quatro casas) é uma das técnicas mais difundidas para caracterização estrutural de uma representação social. No entanto, ocasionalmente os relatos dessa análise não contêm indicações essenciais para que ela seja transparente. $O$ presente texto visa a sistematizar e indicar algumas dessas informações que deveriam estar presentes na descrição de resultados da análise prototípica, discutindo brevemente os prós e contras de algumas opções de realização da análise. Para tanto, é feita uma breve introdução da análise prototípica

1 Endereço para correspondência: R. Octavio Lebarbenchon 69, Florianópolis, SC, CEP 88037-290. E-mail wachelke@yahoo.com (a qual não substitui a leitura das fontes clássicas indicadas no texto) e posteriormente se passa a considerações técnicas acerca da análise.

\section{Apresentação da análise prototípica}

Vergès (1992) desenvolveu uma técnica para caracterizar a estrutura de uma representação social a partir de evocações de palavras. Essa técnica constitui-se de duas etapas: a primeira, chamada análise prototípica, baseia-se no cálculo de frequências e ordens de evocação das palavras, enquanto que uma segunda etapa centra-se na formulação de categorias englobando as evocações e avalia suas frequências, composições e co-ocorrências.

A análise prototípica tornou-se uma das estratégias mais populares para estudar representações sociais, em pesquisas de base (Morin \& Vergès, 1992; Salesses, 2005; Gurrieri, Wolter \& Sorribas, 2007; Wolter, Gurrieri \& Sorribas, 2009) e, especialmente, em pesquisas aplicadas, cujo objetivo não é a contribuição direta para as teorias do pensamento social, mas fundamentalmente a compreensão e diagnósticos ligados a temas sociais, de modo a instrumentalizar intervenções profissionais. Na América Latina, a maior parte das pesquisas sobre estrutura de representações sociais consiste em diagnósticos ligados às áreas da saúde (Tura, 1998; Gomes, Oliveira \& Sá, 2008) e educação (Ferreira \& Souza, 2001; Oliveira, Sá Fischer, Martins \& Teixeira, 2001; Ribeiro \& 
Jutras, 2005; Camargo, Barbará \& Bertoldo, 2007; Naiff, Soares, Azamor \& Almeida, 2008).

Essa popularidade deve-se à relativa simplicidade para conseguir resultados pertinentes a partir de dados pouco estruturados. Caracterizações estruturais ligadas a pesquisas de base ou então aprofundamentos acerca de um diagnóstico representacional vinculado a um grupo geralmente são mais precisos após o emprego de técnicas confirmatórias como as técnicas do "questionamento" (mise en cause) (Moliner, 1989, 2001) e dos esquemas cognitivos de base (Guimelli \& Rouquette, 1992; Rouquette \& Rateau, 1998). No entanto, para estudos com finalidade exploratória a técnica de Vergès dá indicações prováveis de centralidade válidas. Também contribuiu para a difusão da técnica a criação de um programa de computador chamado Evocation (Vergès, Scano \& Junique, 2002), o qual permite efetuar todos os procedimentos de classificação e cálculo exigidos pela análise prototípica.

A análise prototípica parte do pressuposto que os elementos da representação social com importância em sua estrutura são mais prototípicos, isto é, mais acessíveis à consciência (Vergès, Tyzska \& Vergès, 1994). É uma técnica que se aplica a respostas de associação livre, ou seja, frases ou expressões curtas fornecidas a um estímulo indutor, que geralmente é o termo que se refere a um objeto de representação social (Jodelet, 1965; Flament \& Rouquette, 2003).

A análise prototípica geralmente é aplicada sobre dados na forma de palavras ou expressões evocadas por participantes ao lerem ou ouvirem um termo indutor. Assim, restringe-se à expressão da representação social por meio de material verbal recolhido como evocações; cabe apontar, de todo modo, que esse tipo de material não permite uma varredura completa do campo representacional; pesquisas sobre o mascaramento de representações sociais apontam que freqüentemente noções importantes de representações sociais não são evocadas quando há pressões normativas contrárias (Flament, Guimelli $\&$ Abric, 2006). Além disso, o fato de que alguns elementos sejam consensualmente não partilhados também reflete uma dimensão coletiva que delimita o campo da representação.

As pesquisas que empregam análises prototípicas tipicamente solicitam de três a cinco respostas por participante, mas a princípio a técnica poderia ser empregada também com número de respostas diferente, e mesmo sem restrições de quantidade. Nada impede, a rigor, que seja utilizada junto a evocações forçadas, isto é, solicitando-se uma classe ou tipo de palavra específico; porém nesse caso provavelmente os resultados deveriam ser interpretados tendo-se em mente que não são tão espontâneos quanto os gerados pela técnica padrão, e que concluir acerca da estrutura de uma representação social a partir deles pode ser questionável; não obstante, essa alternativa pode ser pertinente para fins diferentes de pesquisa, como, por exemplo, o estudo do processo cognitivo de evocação em si mesmo.

Conforme o procedimento inicial detalhado por Vergès em 1992 (para uma descrição detalhada em português do procedimento, ver Oliveira, Marques, Gomes e Teixeira, 2005), as respostas fornecidas pelos participantes têm duas de suas coordenadas calculadas: a frequência no corpus do grupo e a ordem média de evocação, isto é, o valor resultante de uma média em que o valor 1 é atribuído para a resposta que é fornecida em primeiro lugar, 2 para a segunda res- posta fornecida pelo participante, e assim por diante; como variante, há a possibilidade de pedir para os participantes realizarem uma classificação de importância, sendo atribuído valor 1 para a resposta mais importante, 2 para a segunda mais importante, etc. (Abric, 2003).

Conforme os valores de suas coordenadas, as palavras ou expressões são então classificadas em "alto" ou "baixo", conforme um valor de corte de referência, diferente para cada uma das duas coordenadas; serão discutidos aspectos técnicos acerca da determinação do valor do ponto de corte na próxima seção do texto. As palavras com frequência alta são aquelas com frequência superior ou igual ao valor de corte de referência para essa dimensão, enquanto que aquelas com valores inferiores situam-se na zona de baixa frequência. A classificação para a coordenada de ordem de evocação é idêntica; cabe ressaltar, porém, que são as palavras com baixas ordens de evocação, lembradas primeiro, que trazem maior interesse. Segundo Flament e Rouquette (2003), a análise prototípica nesse ponto baseia-se no princípio segundo o qual o quanto antes uma pessoa se lembra de uma palavra, maior é a representatividade dessa palavra num grupo formado por pessoas com perfil semelhante; os autores referem-se a esse princípio como lei de Marbe. Assim, os critérios de freqüência e ordem de evocação se complementam e fornecem dois indicadores coletivos para caracterizar a saliência de uma palavra num corpus gerado a partir de um grupo.

$\mathrm{O}$ cruzamento das duas coordenadas, classificadas em valores altos e baixos, gera quatro zonas que caracterizam a tabela de resultados da análise prototípica. A zona do núcleo central compreende palavras com alta frequência e baixa ordem de evocação: ou seja, respostas fornecidas por grande número de participantes e evocadas prontamente. Os elementos do núcleo central das representações sociais têm boa probabilidade de estarem representados por algumas das palavras contidas nessa zona. No entanto, cabe ressaltar que não há uma equivalência imediata entre núcleo central e a zona do núcleo na análise prototípica: esta fornece apenas hipóteses de centralidade, que necessitam de verificação por meio de outras técnicas (Sá, 1996; Abric, 2003; Flament \& Rouquette, 2003). Talvez apenas em casos em que alguns elementos se destaquem muito dos demais, por exemplo com frequências muito maiores que os demais componentes da zona do núcleo, seja possível afirmar diretamente que se trata de um elemento central. De modo complementar, pode ser útil registrar a proporção de ocorrências de cada forma na ordem de evocação 1: um estudo de Wachelke (2008) indica que essa informação está relacionada com um maior valor simbólico dos elementos da representação, característica típica de elementos centrais, e que em alguns casos não há coincidência entre uma alta proporção na ordem 1 e uma baixa ordem média de evocação.

As demais zonas referem-se a elementos que muito provavelmente são periféricos. A zona da primeira periferia inclui as respostas com alta frequência e alta ordem de evocação. São respostas com saliência, mas que indicam elementos secundários da representação (Abric, 2003). Cabe indicar, porém, que alguns autores (Pecora \& Sá, 2008; Sá, Oliveira, Castro, Vetere \& Carvalho, 2009) apontam para a possibilidade de que alguns elementos centrais possam vir a compor a primeira periferia. 
Dentre as respostas com frequências de evocação inferiores ao ponto de corte, há a segunda periferia, que inclui aqueles que são evocados como últimas respostas. Essa zona refere a elementos pouco salientes nas duas coordenadas, e portanto menos interessantes para a estrutura da representação do grupo social, trazendo aspectos mais particularizados. Já as respostas com baixas frequências que são evocadas cedo no discurso formam o que se chama de zona de contraste: são respostas minoritárias que podem indicar duas possibilidades: ou são apenas complementos da primeira periferia, ou indicam a existência de um subgrupo que valoriza consistentemente alguns elementos distintos da maioria, talvez até mesmo com um núcleo central diferente (Abric, 2003).

Em sentido estrito, a análise prototípica é uma convenção de apresentação de dados, não uma análise estatística padrão. Não é um procedimento de cálculo de parâmetros e níveis de significação, mas sim um padrão de organização de informações relativas à evocação de formas verbais de modo sintético. Não há consenso acerca de diversos aspectos dessa organização, ainda que uma leitura atenta dos relatos de pesquisa aponte rapidamente para o emprego mais comum de alguns deles.

\section{Aspectos técnicos referentes à realização da análise e descrição dos resultados}

Após a apresentação das características fundamentais da análise prototípica, é necessário discutir com maior detalhe alguns desses aspectos técnicos que lhe são inerentes, bem como as decisões de procedimento que são implicadas por cada um deles: a quantidade de participantes, o tratamento de equivalência dado às respostas, a definição dos valores mínimos de frequência e dos valores de ponto de corte para as duas coordenadas.

Quanto à quantidade de participantes por amostra, não há indicações precisas acerca de um mínimo para ter resultados válidos. Há casos em que é possível chegar a resultados conclusivos até mesmo com um grupo de 25 participantes (Wolter, Gurrieri \& Sorribas, 2009), no entanto talvez se trate de um caso extremo não praticável com todas as configurações de dados. De modo geral, quanto mais numeroso o grupo de participantes, mais estáveis serão os resultados, afinal uma amostra maior tende a gerar resultados menos suscetíveis de influência pela presença de casos extremos e aproximar-se da realidade observada na população de que é extraída; ou seja, permite estimativas mais confiáveis das ocorrências do fenômeno na população. Por essa razão, as amostras geralmente são maiores, realizadas com cerca de 100 (Gomes, Oliveira \& Sá, 2008) ou 200 (Sá, 1998) participantes.

A respeito do tratamento dado às respostas, alguns autores optam por agrupar respostas por critérios semânticos, classificando-as conforme um significado em comum. Assim, respostas "amigos", "amiga" e "amizade" poderiam ser agrupadas numa categoria intitulada ou pela resposta mais frequente entre elas, ou pela resposta que melhor traduzir a classe geral que reflete o que elas têm em comum. Outra possibilidade é a de realizar agrupamentos somente de palavras que compartilhem o mesmo radical e classe, o que recebe o nome de lematização. Nesse caso, o agrupamento reúne ape- nas respostas masculinas e femininas, e no singular e plural. Dentro do exemplo mencionado, "amigos" e "amiga" seriam agrupadas numa só forma, intitulada segundo a resposta mais frequente dentre elas ou, no caso de frequências iguais, na forma masculina e/ou singular, como convenção. Dentro de uma perspectiva propriamente estrutural, a segunda alternativa é a mais adequada, por duas razões. Primeiramente, evita ambiguidades e divergências a respeito da categorização dos conteúdos (Rouquette \& Rateau, 1998; Flament \& Rouquette, 2003), tornando o processo replicável com maior facilidade. Além disso, segundo Vergès (1992), a análise prototípica baseia-se em uma distribuição de respostas que se aproxima da distribuição de Zipf - trata-se de uma distribuição aplicável ao vocabulário de diversas línguas, que estabelece que a freqüência de palavras num corpus está relacionada com o posto da palavra em termos de freqüência por meio de uma lei de potência inversa com expoente próximo de 1; essa relação implica que algumas formas verbais possuem muitas ocorrências e a grande maioria aparece apenas poucas vezes. A categorização semântica das respostas tende a alterar a distribuição dos dados, aumentando a homogeneidade das freqüências; trata-se de uma distorção que pode enviesar o critério do ponto de corte, a depender do critério escolhido para tal, ou a composição dos quadrantes.

Acerca da composição do quadro de resultados, não há também indicações consensuais acerca da frequência mínima para incluir respostas na apresentação da análise. Nesse caso, o mais importante é indicar a frequência mínima escolhida para cada estudo e a quanto esse valor equivale em relação ao total de participantes. É necessário também relatar a proporção de evocações que é representada no quadro após a exclusão dos termos com frequências inferiores à frequência mínima determinada.

Quanto à definição dos pontos de corte para as duas coordenadas de frequência e ordem de evocação, é importante mencionar que não há uma equação ou critério único utilizado. A literatura indica considerável espaço de variação na escolha dos autores acerca do valor dos pontos de corte. Essa variação é menor no que diz respeito à ordem de evocação; geralmente se adota simplesmente como ponto de corte a mediana do número de evocações, quando este é ímpar. Para 3 evocações, seria 2 o ponto de corte, e para 5 , este tem o valor 3. No caso de uma quantidade par de solicitações de resposta por participante, é necessário calcular a média das ordens de evocação e utilizar o valor resultante como ponto de corte. Outra possibilidade diz respeito a calcular a ordem média de evocações levando em consideração somente as palavras incluídas na análise, isto é, descartando-se as palavras abaixo da freqüência mínima estabelecida; os resultados são ligeiramente diferentes dos obtidos por meio da mediana ou média do total de respostas.

Já no que diz respeito ao ponto de corte relativo a frequência, as variações são mais amplas. O procedimento original proposto por Vergès (1992) é o cálculo da frequência média dentre as respostas, após exclusão das evocações com baixas frequências. Quando é intenção obter um quadro mais equilibrado na distribuição das formas verbais pelos quadrantes, pode ser útil a alternativa de calcular a mediana de freqüência dos termos incluídos na análise (após a exclusão dos termos com freqüências baixas) em vez da média, procedimento 
realizado por Wolter (2008). Outras possibilidades voltadas para os padrões da distribuição de respostas podem ser derivadas a partir de características da técnica, e mostrar-se válidas. Assim, uma alternativa é fixar um ponto de corte referente à proporção de evocações contido na zona de alta frequência (por exemplo, cerca de 30\%), e outra é escolher um ponto em que exista um salto perceptível na continuidade das proporções de evocações (por exemplo, observando-se a distribuição de evocações num gráfico). Por fim, no caso em que há uma ou duas formas com muito mais ocorrências que as demais, a utilização dos critérios já mencionados pode ser pouco útil, por incluir muitas formas na zona de alta frequência, e que ao mesmo tempo destoam muito em termos de ocorrências. Uma boa medida pode ser então adotar como ponto de corte a metade da frequência da forma com mais ocorrências em todo o corpus. Caso a configuração dos quadrantes seja muito diferente com esse critério seja muito diferente da propiciada por meio dos critérios convencionais, talvez seja melhor optar pelo critério alternativo, que força uma diferenciação de algumas formas que se distinguem fortemente das outras e, possivelmente, apresentam fortes indícios de constituírem o núcleo central da representação.

Não parece haver motivos explícitos ou claros para opção por um ou outro critério na inclusão de palavras para análise e determinação dos pontos de corte relativos às coordenadas de freqüência e ordem de evocação. A escolha dos autores por um ou outro procedimento é geralmente realizada por razões particulares, talvez por facilidade de interpretação ou necessidade de ilustrar algum aspecto específico de resultados de pesquisa. Assim, é imprescindível que ao se realizar e relatar uma análise prototípica, sejam fornecidas informações referentes ao tratamento de equivalência das evocações, da distribuição das formas evocadas no corpus e da construção dos quadrantes de análise. Na Tabela 1 indicamos as informações que julgamos necessárias no relato de toda e qualquer análise prototípica. Evidentemente, a opção por critérios alternativos pode ser feita, desde que esses critérios sejam devidamente informados e justificados.

\section{Exemplo de relato de análise prototípica}

De modo a ilustrar os princípios comentados no texto, apresentamos a seguir um exemplo de relato resumido de resultados de análise prototípica. Entre parênteses buscamos evidenciar o tipo de informação da Figura 1 a que se refere cada aspecto do relato. Trata-se de uma análise realizada a partir das respostas de 151 estudantes universitários (tamanho da amostra), dentre os quais 110 mulheres, matriculados no curso de psicologia de uma universidade do norte da Itália, os quais forneceram três respostas de evocação à palavra-estímulo "trabalho"; é um estudo voltado para a exploração inicial da estrutura da representação social sobre trabalho. Os dados foram analisados com o auxílio do programa Evocation.

Sem casos omissos, houve 453 evocações (total de evocações), e uma média geral de frequência de 2,45 (freqüência média das evocações). As evocações foram agrupadas conforme procedimentos de lematização (tratamento de equivalência). A freqüência mínima considerada para inclusão das palavras nos quadrantes foi de 5 , pouco mais de $3 \%$ do tamanho da amostra (freqüência mínima para inclusão no quadro).

Referente às determinações dos pontos de corte para as coordenadas dos quadrantes, foi empregado o critério da mediana nas ordens de evocação; como havia 3 respostas por participante, o valor do ponto de corte foi 2 (critério do ponto de corte da ordem de evocação). Palavras com ordem média de evocação inferiores a 2 foram classificadas como tendo baixa ordem de evocação. Quanto ao critério de freqüência, optou-se por incluir nos quadrantes de alta freqüência uma proporção mínima de $30 \%$ das evocações (critério do ponto

Tabela 1. Informações essenciais para relato de análise prototípica.

Informações referentes ao tratamento de equivalência dado às evocações

- Lematização (redução ao radical), categorização por conteúdo, ou outra possibilidade

Informações referentes à distribuição de palavras

- Tamanho da amostra

- Total de evocações

- $\quad$ Frequência média de evocações

- $\quad$ Frequência mínima das evocações para inclusão no quadro (ex.: proporção do total de participantes; corte de frequência préestipulado...)

Informações referentes aos critérios de construção dos quadrantes

- $\quad$ Ponto de corte para frequência entre as zonas de alta e baixa frequência (ex.: frequência maior que a média das formas incluídas na análise; frequência maior que a mediana das formas da análise; escolha por manter uma proporção pré-determinada de evocações na zona de alta frequência; observação de um "salto" na distribuição de Zipf; metade da frequência da forma com mais ocorrências no corpus...)

- $\quad$ Ponto de corte para ordem média de evocações (ex.: ordem correspondente ao ponto médio das possibilidades de resposta da tarefa de evocação; média da ordem de evocação das formas incluídas na análise...) 
Tabela 2. Análise prototípica referente ao termo indutor "trabalho" $(\mathrm{N}=151)$.

\begin{tabular}{|c|c|c|c|c|c|c|}
\hline & \multicolumn{3}{|c|}{ Ord. méd. evoc. < 2} & \multicolumn{3}{|c|}{ Ord. méd. evoc. $>=2$} \\
\hline & Palavra & Freq. & OME & Palavra & Freq. & OME \\
\hline Freq. $>=11$ & cansaço & 38 & 1,76 & dinheiro & 31 & 2,10 \\
\hline \multirow[t]{3}{*}{$(31,6 \%)$} & comprometim. & 30 & 1,70 & satisfacão & 21 & 2,11 \\
\hline & & & & ganhar & 16 & 2,00 \\
\hline & & & & realização & 11 & 2,18 \\
\hline \multirow[t]{6}{*}{ Freq. $<11$} & salário & 7 & 1,57 & paixão & 8 & 2,00 \\
\hline & atividade & 7 & 1,71 & obrigação & 5 & 2,00 \\
\hline & profissão & 7 & 1,71 & segurança & 5 & 2,00 \\
\hline & responsibilidade & 7 & 1,86 & prazer & 5 & 2,20 \\
\hline & fragilidade & 5 & 1,00 & vida & 5 & 2,60 \\
\hline & escritório & 5 & 1,40 & & & \\
\hline
\end{tabular}

de corte para freqüência); no caso da presente análise, isso se deu a partir da freqüência 11; palavras com essa frequência ou valores superiores corresponderam a $31,6 \%$ do total de evocações.

A Tabela 2 apresenta os resultados da análise prototípica mencionada. A análise foi realizada integralmente em italiano, mas para a apresentação dos resultados foi realizada tradução das palavras incluídas no quadro para o português. As duas palavras que provavelmente se referem a elementos centrais da representação social sobre trabalho são "cansaço" e "comprometimento", ambas com altas freqüências (maiores que 11) e ordens médias de evocação inferiores a 2. Ou seja, o conhecimento compartilhado por esses estudantes italianos caracteriza-se por conceber um trabalho como uma atividade que necessariamente é cansativa e exige dedicação. O elemento "dinheiro", apesar de não estar na zona do núcleo central por ter uma ordem de evocação mais alta que o ponto de corte, também deveria ser analisado em pesquisas futuras, por ter alta freqüência; note-se que a palavra "salário" também tem significado semelhante e é lembrada mais prontamente, o que sugere que aspectos ligados a remuneração em dinheiro sejam investigados posteriormente com técnicas confirmatórias. É pertinente apontar o contraste dos aspectos de obrigação e esforço ligados ao trabalho, vistos como essenciais, com "realização", "paixão", "satisfação" e "prazer", todos periféricos. Trabalhar, para os participantes dessa pesquisa, está mais próximo de "trabalhar duro para obter uma remuneração" que de "trabalhar para obter realização pessoal".

\section{Conclusão}

Mesmo na ausência de um consenso acerca das indicações para relato de alguma análise científica, a principal diretriz a se levar em conta é provavelmente a transparência acerca dos critérios escolhidos para orientar a análise, de modo que todas as informações necessárias para caracterizar os resultados sejam relatadas. Somente com o fornecimento dessas informações, os resultados tornam-se plenamente pertinentes para a comunidade acadêmica que se ocupa do estudo das representações sociais, possibilitando o entendimento e a avaliação dos esforços de pesquisa realizados de modo integral. Sendo a objetividade uma característica sempre desejável de estudos científicos, e ao se considerar que ela pode ser atingida por meio desse tipo de indicação, ressaltamos a importância de que os resultados de pesquisa sejam transparentes, para a construção de um corpo de conhecimentos sólido e replicável.

Por fim, cabe enfatizar que a análise prototípica é apenas uma das diversas técnicas de caracterização existentes para as representações sociais, e sublinhar suas limitações. É um recurso prático mas que permite apenas a identificação de compartilhamento acerca de elementos da representação por meio de padrões de consenso intragrupo quantitativo mais evidentes, no que diz respeito a freqüências superiores ao ponto de corte estabelecido. Assim, interessa-se explicitamente às palavras invariantes para o grupo. As palavras que compõem quadrantes periféricos, especialmente as de baixas freqüências, apontam para aspectos em que existe variabilidade no grupo, mas são elementos colocados em segundo plano pela análise; na etapa em que se mostra útil para pesquisas exploratórias, a análise prototípica contribui ao identificar os elementos menos suscetíveis a variações desse tipo; um estudo da variabilidade em si seria melhor proporcionado com o auxílio de outras técnicas como a análise de correspondências aplicada ao estudo das representações sociais (Doise, Clemence \& Lorenzi-Cioldi, 1992), que busca precisamente identificar tomadas de posições específicas frente a princípios organizadores compartilhados, por meio de associações de variáveis nominais. Quanto à variabilidade intergrupo acerca de uma representação social, a análise prototípica pode fornecer informações úteis, mas somente se for realizado um estudo comparativo das zonas dos núcleos centrais de grupos diferentes acerca do mesmo objeto; considera-se que dois grupos têm representações diferentes quando seus núcleos centrais são diferentes. Ou seja, a análise de um grupo isolado pouco pode ajudar para entender o fenômeno das representações sociais: é necessário sempre ter em vista o contexto social em que grupo e objeto estão inseridos, para dar conta de uma compreensão satisfatória do fenômeno. 


\section{Referências}

Abric, J.-C. (2003). La recherche du noyau central et de la zone muette des représentations sociales. In J.-C. Abric (Ed.), Méthodes d'étude des représentations sociales (pp. 59-80). Ramonville- Saint Agne : Érès.

Camargo, B.V., Barbará, A., \& Bertoldo, R.B. (2007). Concepção pragmática e científica dos adolescentes sobre a AIDS. Psicologia em Estudo, 12, 277-284.

Doise, W., Clemence, A., \& Lorenzi-Cioldi. F. (1992). Représentations sociales et analyse des données. Grenoble: PUG.

Ferreira, A. S., \& Souza, L. (2001). Representação social da escola segundo aluno superdotados. Arquivos Brasileiros de Psicologia, 53(4), 31-52.

Flament, C., Guimelli, C., \& Abric, J.-L. (2006). Effets de masquage dans l'expression d'une représentation sociale. Cahiers Internationaux de Psychologie Sociale, 69, 15-31.

Flament, C., \& Rouquette, M.-L. (2003). Anatomie des idées ordinaires. Paris: Armand Colin.

Gomes, A. M. T., Oliveira, D. C., \& Sá, C. P. (2008). As representações sociais do Sistema Único de Saúde no município do Rio de Janeiro, Brasil, segundo a abordagem estrutural. Revista Latino-americana de Enfermagem, 16, 122-129.

Guimelli, C., \& Rouquette, M.-L. (1992). Contribution du modèle associatif des schèmes cognitifs de base à l'analyse structurale des représentations sociales. Bulletin de Psychologie, 45, 196-202.

Gurrieri, C., Wolter, R. P., \& Sorribas, E. (2007). L'implication personnelle: un outil psychosocial pour comprendre le lien population-objet. Psicologia em Estudo, 12, 423-432.

Jodelet, F. (1965). L'association verbale. In P. Fraisse \& J. Piaget (Eds.), Traité de psychologie expérimentale, VII : Langage, communication et décision (pp. 93-137). Paris : PUF.

Moliner, P. (1989). Validation expérimentale de l'hypothèse du noyau central des représentations sociales. Bulletin de Psychologie, 41, 759-762.

Moliner, P. (2001). Consensus manifestes, consensus latents et consensus illusoires. Cahiers Internationaux de Psychologie Sociale, 49, 114-122.

Morin, M., \& Vergès, P. (1992). Enquête sur une représentation en voie d'émancipation: le sida pour les jeunes. Cahiers Internationaux de Psychologie Sociale, 15, 46-75.

Naiff, L. A. M., Soares, A. B., Azamor, C. R., \& Almeida, S. A. (2008). Ensino fundamental e médio: aspectos psicossociais do bom desempenho profissional. Arquivos Brasileiros de Psicologia, 60(3), 64-76.

Oliveira, D. C., Marques S. C., Gomes, M. T., \& Teixeira, M. C. T. V. (2005). Análise de evocações livres: uma técnica de análise estrutural das representações sociais. In A. S. P. Moreira, B. V. Camargo, J. C. Jesuíno, \& S. M. Nóbrega (Eds.), Perspectivas teórico-metodológicas em representações sociais (pp.573603). João Pessoa, UFPB.

Oliveira, D. C., Sá, C. P., Fischer, F. M., Martins, I. S., \& Teixeira, L. R. (2001). Futuro e liberdade: O trabalho e a instituição escolar nas representações sociais de adolescentes. Estudos de Psicologia (Natal), 6, 245-258.
Pecora, A. R., \& Sá, C. P. (2008). Memórias e representações sociais da cidade de Cuiabá, ao longo de três gerações. Psicologia Reflexão e Crítica, 21, 319-325.

Ribeiro, M. L., \& Jutras, F. (2005). Representações sociais de professores sobre afetividade. Estudos de Psicologia (Campinas), 23, 39-45.

Rouquette, M.-L., \& Rateau, P. (1998). Introduction à l'étude des représentations sociales. Grenoble: PUG.

Sá, C. P. (1996). Núcleo central das representações sociais. Petrópolis: Vozes.

Sá, C. P. (1998). A representação social da economia brasileira antes e depois do "Plano Real". In A. S. P. Moreira \& D. C. Oliveira (Eds.), Estudos interdisciplinares de representação social (pp. 49-69). Goiânia: AB.

Sá, C. P., Oliveira, D. C., Castro, R. V., Vetere, R., \& Carvalho, R. V. C. (2009). A memória histórica do regime militar ao longo de três gerações no Rio de Janeiro: sua estrutura representacional. Estudos de Psicologia (Campinas), 26, 159-171.

Salesses, L. (2005). Rôle du niveau de connaissance dans le processus de structuration d'une représentation sociale. Cahiers Internationaux de Psychologie Sociale, 66, 25-42.

Tura, L. F. R. (1998). Aids e estudantes: a estrutura das representações sociais. In D. Jodelet \& M. Madeira (Eds.), Aids e representações sociais: à busca de sentidos (pp. 121154). Natal: UFRN.

Vergès, P. (1992). L'évocation de l'argent: une méthode pour la définition du noyau central de la représentation. Bulletin de Psychologie, 45, 203-209.

Vergès, P.,; Scano, S., \& Junique, C. (2002). Ensembles de programmes permettant l'analyse des évocations. Aix en Provence: Université de Provence.

Vergès, P., Tyszka, T., \& Vergès, P. (1994). Noyau central, saillance et proprietés structurales. Papers on Social Representations, 3, 3-12.

Wachelke, J. (2008). Relationship between response evocation rank in social representations associative tasks and personal symbolic value. International Review of Social Psychology, 21, 113-126.

Wolter, R. P. (2008). Pensée sociale et situations de crise: le rôle des nexus dans l'implication personnelle et les modes de raisonnement. Tese de Doutorado, Université Paris Descartes, Paris.

Wolter, R. P., Gurrieri, C., \& Sorribas, E. (2009). Empirical illustration of the hierarchical organisation of social thought: a domino effect? Interamerican Journal of Psychology, 43, 1-11.

Recebido em 15.01.2010

Primeira decisão editorial em 08.11.2010

Versão final em 26.11.2010

Aceito em 01.02.2011 